\title{
Using Cellular Probabilistic Self-Organizing Map in Borrowing Channel Assignment for Patterned Traffic Load
}

\author{
SITAO WU^, TOMMY W. S. CHOW and KAI TAT NG \\ Department of Electronic Engineering, City University of Hong Kong, Hong Kong. \\ e-mail:stwu@ee.cityu.edu.hk
}

\begin{abstract}
The fast growing cellular mobile systems demand more efficient and faster channel allocation techniques. Borrowing channel assignment (BCA) is a compromising technique between fixed channel allocation (FCA) and dynamic channel allocation (DCA). However, in the case of patterned traffic load, BCA is not efficient to further enhance the performance because some heavy-traffic cells are unable to borrow channels from neighboring cells that do not have unused nominal channels. The performance of the whole system can be raised if the short-term traffic load can be predicted and the nominal channels can be re-assigned for all cells. This paper describes an improved BCA scheme using traffic load prediction. The prediction is obtained by using the short-term forecasting ability of cellular probabilistic self-organizing map (CPSOM). This paper shows that the proposed CPSOM-based BCA method is able to enhance the performance of patterned traffic load compared with the traditional BCA methods. Simulation results corroborate that the proposed method delivers significantly better performance than BCA for patterned traffic load situations, and is virtually as good as BCA in the other situations analyzed.
\end{abstract}

Key words. borrowing channel assignment (BCA), cellular probabilistic SOM (CPSOM), dynamic channel allocation (DCA), fixed channel allocation (FCA), patterned traffic load, self-organizing map (SOM)

\section{Introduction}

The technology and applications of cellular mobile systems have been growing with an extraordinary rate. The $3 \mathrm{G}$ mobile technology is freshly released for the everincreasing demand on mobile communication. And universal mobile telecommunications system (UMTS) is a third-generation system designed to support higher data transmission rate and better quality of service for mobile users. In order to satisfy the increasing number of users with the very limited spectrum resource, research on utilizing the radio spectrum efficiently has become an exigent issue.

In the UMTS terrestrial radio access network (UTRAN), a geographical region is spatially divided into a number of cells. A base station, referred as Node B, is established in each cell and user equipments (UE's) communicate with each other through Node B's. To access the UMTS services, a UE has to communicate with

${ }^{\star}$ Corresponding author. 
cells (Node B's) over the air interface. There is, however, certain inevitable interference across channels. First, a UE in a cell uses a channel that may be currently occupied by another UE in a nearby cell (co-channel inference). Second, a UE in a cell may use a channel that is adjacent to another channel currently occupied by another UE in an adjacent cell (adjacent channel inference). Third, a UE in a cell may use a channel that is near another channel currently used by another UE in the same cell (co-site interference).

Several types of channel assignment strategies have been proposed in different literatures. They can be mainly classified into three types [1]: (1) Fixed channel allocation (FCA); (2) Dynamic channel allocation (DCA); (3) Hybrid channel allocation (HCA). In FCA [2], a set of nominal channels is permanently assigned to each cell and there is a tolerable level of interference for these assigned channels. The permanent channel assignment is performed according to the estimated peak traffic load in each cell. If no unused nominal channels in a cell are available, a new call in that cell will be blocked. It has been shown that FCA is a generalized graph coloring problem [3] and is therefore NP-hard. Many methods have been proposed to provide the solution of FCA, i.e., neural-network-based methods [4-7], simulated annealing methods [8, 9], Tabu search methods [10, 11], genetic algorithm [12], Ants algorithm [13], graph coloring methods [3, 14], Viterbi-like algorithm [15, 16], other heuristic algorithm [17], and hybrid algorithm [18], etc. However, more calls will be blocked when the current traffic load varies greatly and deviates from the estimated peak traffic load considerably. Thus the inflexibility for nonstationary traffic load is a disadvantage of FCA.

Contrary to FCA, DCA dynamically allocates channels in a real-time fashion. DCA assigns channels in a way according to the current traffic demand. In DCA, each channel can be a candidate nominal channel of a cell. This enables the nominal channels of each cell changing dynamically in DCA. The whole channel utilization in DCA delivers better result than that in FCA under low and normal traffic load scenario. Some examples of centralized DCA are neural-network-based methods [19-21], etc. It is, however, worth noting that DCA is not superior to FCA under heavy traffic condition and the computation of DCA is more complex and time-consuming.

HCA is a compromise of FCA and DCA. Some examples of HCA are the borrowing channel assignment (BCA) methods [22-27]. In BCA, all the nominal channels are initially allocated by a FCA method. When new calls arrive with all the nominal channels in a cell being used, the cell borrows unused nominal channels from its neighboring cells under the constraint of tolerable interference. Otherwise, the new calls will be blocked. However, when a nominal channel is borrowed from some of its neighboring cells, several other cells can be affected and cannot use that channel due to the co-channel interference [25]. Thus, the total number of the available nominal channels in all cells decreases amid the borrowing process, because there are certain nominal channels prohibited from being used. Since BCA does not collect system-wide information, the computation of $\mathrm{BCA}$ is relatively simpler and more 
efficient than that of DCA. Although BCA is a heuristic method, it is very suitable and appealing to real-time (or online) channel allocation.

The aforesaid BCA methods are based on FCA. The assumption of FCA is that the real peak traffic does not deviate much from the estimated peak traffic for which the network has been designed. In certain situations, many cells with heavy traffic cluster together. The inner cells inside the heavy-traffic cluster are not able to borrow channels because their neighboring cells are short of unused nominal channels due to the heavy traffic loading. Meanwhile, other cells outside the heavy-traffic cluster may have sufficient unused nominal channels due to the relatively light or medium traffic loading. In addition, the number of light-or-mediumtraffic cells is less than that of heavy-traffic cells. This situation, called patterned traffic load because these heavy-traffic cells form meaningful patterns, is of major interest to us. The channels are not fully utilized because the initial channel assignment of BCA may not be suitable for future traffic load. Apparently, we are able to enhance the performance of BCA for patterned traffic load if the short-term traffic load can be forecasted and the channel assignment can be adjusted periodically. In this letter, an improved BCA method based on cellular probabilistic self-Organizing map (CPSOM) [28], is proposed to predict the short-term traffic load and thereafter re-assign all the nominal channels for all cells. CPSOM is an extended self-organizing map (SOM) [29] aiming at data quantization and topology preservation. When the traffic load does not vary too rapidly, traffic prediction can be obtained with a respectable level. As a result of prediction and re-assignment, the heavy-traffic cells can obtain more nominal channels while the numbers of the nominal channels in other light-or-medium-traffic cells are decreased accordingly. Compared with BCA, the performance of the heavy-traffic cells without neighboring light-or-medium-traffic cells is raised by the proposed method due to the increased number of nominal channels. Since other heavy-traffic cells can still borrow channel from their neighboring light-or-medium-traffic cells, the performance of them delivered by the proposed method is similar to that of BCA. The performance of the light-or-medium-traffic cells is decreased by the proposed method due to the reduced number of nominal channels. When the performance of the whole system is evaluated, the heavy-traffic cells are associated with large weights and the light-or-medium-traffic cells are associated with small weights. Furthermore, the number of the heavy-traffic cells is more than that of the lightor-medium-traffic cells for patterned traffic load. Therefore, the satisfaction probability of the whole system, i.e., the total number of all successfully accepted calls without dropping over that of all calls, delivered by the proposed method is higher than that delivered by the traditional BCA methods under patterned traffic load. For other traffic situations, if the re-assignment is performed after prediction, the satisfaction probability delivered by the proposed method is similar to that by the traditional BCA methods. Therefore the re-assignment will not be performed if other traffic situations are detected in the proposed method. Although CPSOM is used directly for traffic prediction and the original BCA methods are not altered, 
the proposed method can deliver significantly improved performance than BCA under patterned traffic load situation, and is virtually as good as BCA in the other situations analyzed. Furthermore, if other BCA methods are used with CPSOMbased prediction and re-assignment afterwards, the results will be similar.

The content of this letter is organized as follows. In Section 2, BCA is briefly reviewed and the channel utilization by BCA for patterned traffic load is explained. In Section 3, the CPSOM algorithm and the whole CPSOM-based BCA method are described in detail. Simulation results are provided in Section 4. Finally, conclusion is drawn in Section 5.

\section{BCA and Patterned Traffic Load}

There are several different versions of BCA: simple borrowing (SB) strategy [22], hybrid assignment (HA) strategy [23], borrowing with channel-ordering (BCO) strategy [24], borrowing with directional channel-locking (BDCL) strategy [25], two-phase BCA (TPB) strategy [26], heuristic BCA (HB) [27], etc. SB is the simplest $\mathrm{BCA}$ and is suitable for light and medium traffic load. The blocking probability is increased under heavy traffic for SB [25]. In HA, the nominal channels in a cell are divided into two parts. The nominal channels in a part are used for the local cell, whilst the nominal channels in the other part can be borrowed by other cells. The ratio of numbers of channels between the two parts in the cell is set $a$ priori. $\mathrm{BCO}$ improves $\mathrm{HA}$ by automatic determination of the ratio according to traffic load. BDCL further improves BCO by reducing the number of locked cells. TPB is a two-phase BCA that achieves lower blocking probability than BDCL. In $\mathrm{HB}$, channels are borrowed according to three heuristics. But HB does not outperform BDCL and its computation is time-consuming.

In all of the above BCA methods, the channels are initially allocated by a FCA method. Under patterned traffic load, the channel utilization is not efficient as mentioned in Section 1. This view is demonstrated in Figure 1, where Figure 1(a) shows a cellular system with 16 cells and Figure 1(b) shows the total number of the current busy calls and new calls for each cell at a time. Suppose that BCA initially allocates 10 nominal channels for each cell. Therefore from Figure 1(b), cells $1,2,3,5$ and 6 have medium traffic and have some unused nominal channels. The other cells cluster together with heavy traffic. The inner cells $8,11,12,13$, 14,15 and 16 in the cluster do not have neighboring cells with medium traffic and cannot borrow channels from their neighboring cells since their neighboring cells have also heavy traffic and run out of unused nominal channels. The numbers of the heavy-traffic and medium-traffic cells are 11 and 5, respectively. This is the patterned traffic load mentioned before. Since some unused nominal channels are still available in the medium-traffic cells and some heavy-traffic cells have no channels to borrow, all the nominal channels are not fully utilized. Our proposed method overcomes this problem by utilizing more channels through a traffic-prediction technique by CPSOM-based prediction and re-assignment after prediction. The basic principle 

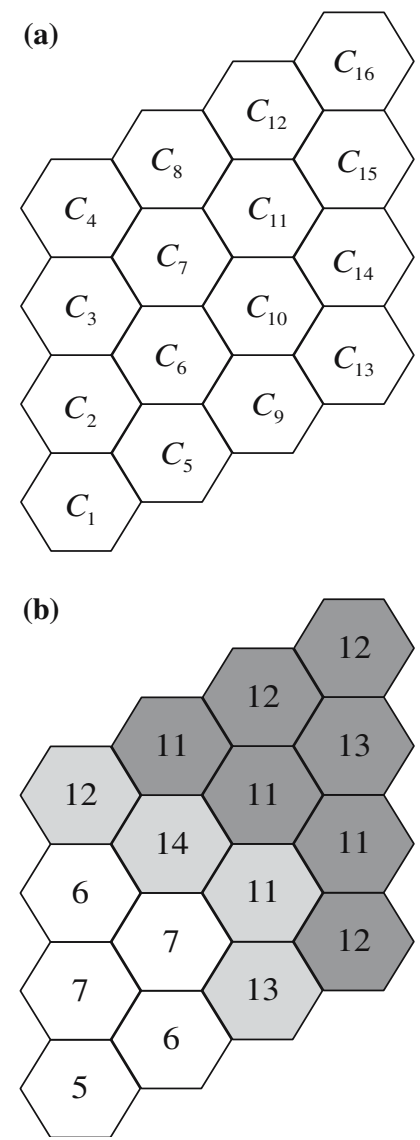

Figure 1. A cellular system with 16 cells. (a) The symbol $C_{i}$ in a cell denotes the $i$ th cell; (b) The number in a cell denotes the total number of the current busy calls and new calls in that cell at a time. Medium-traffic cells are in the white area. Heavy-traffic cells without neighboring medium-traffic cells are in the heavy gray area and heavy-traffic cells with neighboring medium-traffic cells are in the light gray area.

of the proposed method is that the heavy-traffic cells have more nominal channels and light-or-medium-traffic cells have less for patterned traffic load.

\section{The CPSOM Algorithm and the CPSOM-Based BCA Method}

\subsection{THE CPSOM ALGORITHM}

When a short-term traffic load can be roughly predicted, all cells can be re-assigned in a way that heavy-traffic cells are given more nominal channels, and light-ormedium-traffic cells are given less ones. The traffic-prediction technique adopted in this letter is the online CPSOM algorithm [28], which is an improvement of the traditional SOM algorithm [29]. CPSOM quantizes the latest input data and 
reflects the future input distribution if the distribution does not vary fast. Thus CPSOM can be used for the short-term traffic prediction in the proposed method.

The traditional SOM algorithm has been widely used in many areas such as pattern recognition, biological modeling, data compression, signal processing, and data mining. SOM is well known for its ability of performing vector quantization and preserving topology. The neurons of SOM can be considered quantized vectors embedded into the input data. In this letter, we use the weight vector of a neuron, which reflects one of the average traffic loads in history, to predict the future traffic if the traffic in the system does not vary fast. However, the traditional SOM algorithm cannot be used in online environments, where the input data come in endlessly and dynamically, because the learning rate of SOM will decrease to nearly zero and it has no effect on training thereafter. CPSOM improves SOM and is very suitable for online environments. The details of CPSOM are described in [28]. The following briefly describes the CPSOM algorithm:

Step 1: Initialize weights $\left\{w_{1}, \ldots, w_{L}\right\}$ of all the $L$ neurons to be random vectors, calculate the neighborhood function $h_{i j}$ between neuron $i$ and $j$, where $h_{i j}$ satisfies $\sum_{j=1}^{L} h_{i j}=1$, and set the start values $\beta=\beta^{\text {start }}(>0)$ and $\gamma=\gamma^{\text {start }}(>1)$, where $\beta$ is a parameter of inverse temperature used for simulated annealing and $\gamma$ is used to re-train CPSOM in online environments.

Step 2: Select an input $x$ sequentially from dynamic data sets.

Step 3: Find the probability assignments and adapt weights for all neurons:

$E$ step: $P_{i}(x(t))=\frac{\exp \left(-\beta \sum_{j=1}^{L} h_{i j} D\left(x(t), w_{j}\right)\right)}{\sum_{n=1}^{L} \exp \left(-\beta \sum_{j=1}^{L} h_{n j} D\left(x(t), w_{j}\right)\right)}, \quad i=1, \ldots, L$,

$M$ step: $w_{i}(t)=w_{i}(t-1)+\frac{1}{B_{i}(t)} \sum_{j=1}^{L} h_{i j} P_{j}(x(t))\left(x(t)-w_{i}(t-1)\right)$,

$$
i=1, \ldots, L,
$$

where $B_{i}(t)=B_{i}(t-1)+\sum_{j=1}^{L} h_{i j} P_{j}(x(t)), \quad i=1,2, \ldots, L$. In the $E$ step, $P_{i}(x(t))$ is the assignment probability of neuron $i$ upon the input $x(t)$, $D\left(x(t), w_{j}\right)$ is the quantization error between the input $x(t)$ and the weight $w_{j}$ of neuron $j$, defined by $D\left(x(t), w_{j}\right)=\frac{1}{2}\left\|x(t)-w_{j}\right\|^{2}$.

Step 4: When the current iteration $t$ is an integer multiple of a parameter $\lambda_{1}$, if $\beta<\beta^{\text {final }}$, increase $\beta$ by $\beta \leftarrow \beta+\Delta_{1}$, where $\Delta_{1}(>0)$ is an incremental factor. Otherwise, $\beta=\beta_{\text {final }}$.

Step 5: When the current iteration $t$ is an integer multiple of a parameter $\lambda_{2}$, decrease $B_{i}(t)$ by $B_{i}(t)=B_{i}(t) / \gamma, i=1,2, \ldots, L$, where $\gamma \leftarrow \gamma-\Delta_{2}(\gamma \geqslant$ $\left.1, \Delta_{2}>0\right)$ is a factor shrinking to a final value $\gamma^{\text {final }}=1$. This procedure enables the algorithm to re-train the network with a higher learning rate after certain iterations to avoid being trapped in local minima. In fact, $\gamma$ can be considered as a forgetting factor to reduce the weight of the old 
input data contained in the $B_{i}(t)^{\prime} s$. When CPSOM is used for online environments, if the current iteration $t$ is an integer multiple of a parameter $\lambda_{3}\left(\lambda_{3} \gg \lambda_{2}\right.$ and $\lambda_{3}$ is an integer multiple of $\left.\lambda_{2}\right)$, set $\gamma=\gamma^{\text {start }}$. This procedure ensures the learning algorithm to track online environments.

Step 6: Go to Step 2 for online environments.

When CPSOM is used for prediction, the weight vectors are periodically updated every minute by Step 3 . The $i$ th item $x_{i}(t)$ in the input vector $\left[x_{1}(t), \ldots, x_{N}(t)\right]^{T}$ in a cellular system with $N$ cells at time $t$ is the number of new calls in cell $i$ during the latest one minute before time $t$. The weight vector of neuron $i$ in CPSOM is represented by $\left[w_{i 1}, \ldots, w_{i N}\right]^{T}$.

\subsection{THE CPSOM-BASED BCA METHOD}

After CPSOM has been run in a cellular system for some time, e.g., at time $t$, the weight vector of a neuron can represent a traffic distribution that may reflect the real traffic in the system. The weight vector can then be used to represent the future traffic trend if the traffic in the system does not change fast and can be roughly predicted by CPSOM. After prediction and patterned traffic load happens at that time, all nominal channels can be re-assigned by a FCA method to give a better channel utilization. The total number of all the nominal channels after re-assignment is the same as that before re-assignment. For example, the number of all the nominal channels is $M$. The numbers of nominal channels for all $N$ cells before re-assignment are $\left\{N o_{1}, \ldots, N o_{N}\right\}$, where $N o_{i}$ is the number of nominal channels for cell $i$ and $\sum_{i=1}^{N} N o_{i}=M$. A nearest neuron, e.g., neurons $i$, to the current input $x(t)$ is found. The weight vector of neuron $i$ records one of the average traffics in the past and can be used to predict the future traffic trend if the traffic does not vary fast.

After the weight vector $W_{i}=\left[w_{i 1}, \ldots, w_{i N}\right]^{T}$ of neuron $i$ is selected, it is transformed to an integer vector by $W_{i}^{\prime}=\left[w_{i 1}^{\prime}, \ldots, w_{i N}^{\prime}\right]^{T}$ where $M-N<$ $\sum_{j=1}^{N} w_{i j}^{\prime}=M^{\prime}<M$ and $w_{i j}^{\prime}=\left\lfloor\left(M w_{i j}\right) /\left(\sum_{j=1}^{N} w_{i j}\right)\right\rfloor, j=1, \ldots, N$, where $\lfloor x\rfloor$ is a function that takes the integer part from a real number $x$. After the transformation of the weight vector $W_{i}$ to $W_{i}^{\prime}$, several nominal channels are added to cells such that the number of all the nominal channels after re-assignment is the same as that before re-assignment. Therefore, $\left(M-M^{\prime}\right)$ channels are added to some cells by the following steps.

Step 1: Sort all the elements $\left\{w_{i 1}, \ldots, w_{i N}\right\}$ of $W_{i}$ in a descending order. If two elements are equal, sort them randomly. The final sorted elements are $\left\{w_{i k_{1}}, \ldots, w_{i k_{N}}\right\}$ such that $w_{i k_{1}} \geqslant w_{i k_{2}} \geqslant \cdots \geqslant w_{i k_{N}}$, where $k_{m} \in$ $\{1, \ldots, N\}, m=1, \ldots, N$.

Step 2: Firstly, generate a new integer weight vector $W_{i}^{\prime \prime}=\left[w_{i 1}^{\prime \prime}, \ldots, w_{i N}^{\prime \prime}\right]^{T}$ such that $w_{i j}^{\prime \prime}=w_{i j}^{\prime}, j=1, \ldots, N$. Secondly, $\left(M-M^{\prime}\right)$ channels are added to 
some cells:

$$
\begin{array}{r}
\text { for } m=1 \text { to } M-M^{\prime} \\
w_{i k_{m}}^{\prime \prime}=w_{i k_{m}}^{\prime \prime}+1
\end{array}
$$

end

Finally, the new transformed weight vector $W_{i}^{\prime}=\left[w_{i 1}^{\prime \prime}, \ldots, w_{i N}^{\prime \prime}\right]^{T}$ for re-assignment satisfies $\sum_{j=1}^{N} w_{i j}^{\prime \prime}=M$.

The re-assignment is performed by firstly using a FCA method. The computation time by a FCA method is supposed to be $\delta$. At time $t+\delta$, the current busy and new calls in all cells are re-assigned to the new allocated nominal channels. The number of nominal channels of cell $j$ for the re-assignment is just $w_{i j}^{\prime \prime}$. During the re-assignment, some busy calls may be dropped out. If only blocking probability is considered, the dropped calls may not be considered for the proposed method. Therefore the later comparison among the proposed method and other methods is based on satisfaction probability, which is defined as the ratio of the number of all accepted calls without dropping over that of all calls. The higher the satisfaction probability is, the better the performance is.

How can we detect patterned traffic load? Firstly, the satisfaction probability of the whole system by BCA in the past is between about $65 \%$ and $97 \%$ empirically, which means that there are heavy traffic in some cells. Satisfaction probability below $65 \%$ means that all cells can have heavy traffic whilst that above $97 \%$ means that all cells can have light or medium traffic. Secondly, the mean value $\mu$ of $\left\{w_{i 1}, \ldots, w_{i N}\right\}$ is computed. If $w_{i j}$ is larger than or equal to $\mu$, cell $j$ has heavy traffic. Otherwise cell $j$ has light or medium traffic. Thus all cells can be divided into two classes: light-or-medium-traffic cells (class $A$ ) and heavy-traffic ones (class $B$ ). When more than $60 \%$ of all cells are class $B$ and more than $40 \%$ class $B$ cells have no neighboring class $A$ cells empirically, more new calls will be rejected by BCA in the heavy-traffic cells without neighboring light-or-medium-traffic cells. This situation is called patterned traffic load. The traffic loads shown in Figure 2 (a) and (b) are patterned traffic loads whilst those shown in Figure 2(c) and (d) are not.

Finally, the whole CPSOM-based BCA method is described as follows.

Step 1: Initially use a FCA method to allocate nominal channels for each cell in advance.

Step 2: Initialize the CPSOM network.

Step 3: Set the current time $t=0$ (second).

Step 4: Allocate channels for new calls at time $t$ and borrow channels by a BCA method if possible.

Step 5: When the current iteration $t$ is an integer multiple of 60 , i.e., one minute later, an input vector $x(t)$ is formed and is fed into the CPSOM network and train the CPSOM network online. 

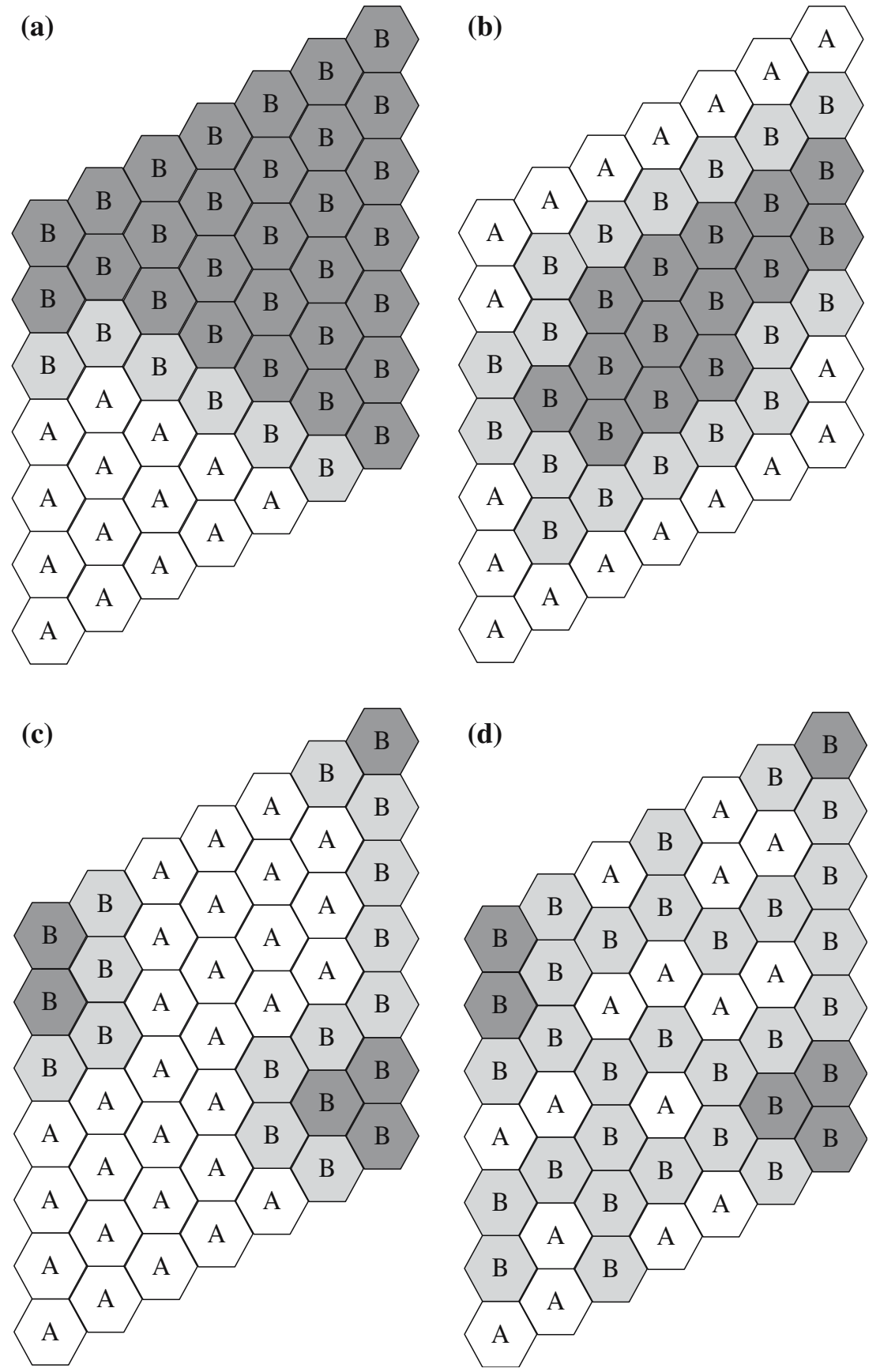

Figure 2. Traffic distribution of two classes $A$ and $B$. Light-traffic cells (class $A$ ) are in the white area. Heavy-traffic cells (class $B$ ) without neighboring light-traffic cells are in the heavy gray area and heavy-traffic cells (class $B$ ) with neighboring light-traffic cells are in the light gray area. (a)-(b) are patterned traffic loads; (c)-(d) are not patterned traffic loads. 
Step 6: When the current iteration $t$ is an integer multiple of a parameter $\tau(\tau \gg$ $60 \mathrm{~s}$ ), the nearest neuron $i$ from $x(t)$ is found. After patterned traffic load is detected and $W_{i}$ is transformed to $W_{i}^{\prime \prime}$, use a FCA method to allocate new nominal channels in background. Otherwise, go to Step 8.

Step 7: If FCA is finished, re-assign the current busy and new calls to the new nominal channels for all cells.

Step 8: $t=t+1$, go to Step 4 .

\section{Simulation Results}

The simulations were done on a cellular system shown in Figure 3(a). There are totally 16 cells and 140 channels available in the system. The call arrivals are supposed to follow a Poisson process with the mean arrival rate of $\lambda$ (calls $/ \mathrm{min}$.). The call duration is an exponentially distributed statistical process with mean $\bar{x}$ (min.). The load offered to the system is $\rho=\lambda \cdot \bar{x}$.

In this letter, adjacent channel interference requires that any two channels assigned to two adjacent cells must be at least 2 channels apart from each other. Co-site interference requires that any two channels assigned to the same cell must be at least 2 channels apart from each other. The reuse distance to avoid co-channel interference is set to three cell units. The initial channel allocation is set by a FCA method in [7]. For estimated peak traffic load in advance, each cell is assumed to be allocated with 10 nominal channels in the simulation. The BCA method used in the proposed method is SB. Three examples of different types of traffic loads were used in the simulations. Note that the three traffic loads are temporally stationary. All the simulations were simulated for $5 \mathrm{~h}$. The performance of channel allocation is evaluated by satisfaction probability.

For the CPSOM algorithm, a $3 \times 3$ network size was used. Some settings of parameters in CPSOM are the following: $L=9, \beta^{\text {start }}=50.0, \beta^{\text {final }}=200, \gamma^{\text {start }}=$ 8.0, $\Delta_{1}=0.1, \Delta_{2}=0.1, \lambda_{1}=200, \lambda_{2}=200, \lambda_{3}=2000$. The inputs to the CPSOM network are new calls in the latest one minute before the current time and is normalized by dividing it over a constant 10 . In the proposed method, $\tau=3600 \mathrm{~s}(1 \mathrm{~h})$.

The first simulation was done by using spatially uniform traffic in the system. The mean arrival rate $\lambda$ is 1.5 calls $/ \mathrm{min}$. The call duration $\bar{x}$ is $3 \mathrm{~min}$. FCA, SB and the proposed method were used in the allocation. Since the chance of patterned traffic load is little, patterned traffic load was not detected in Step 6 of the proposed method. But here we canceled the detection of patterned traffic load in the proposed method for later comparison and the re-assignment was performed. The first channel re-assignment from the beginning by the proposed method is shown in Figure 4(a). The new assignment is almost the equal assignment like the initial assignment. The other channel re-assignment at other time is similar to the situation shown in Figure 4(a). The increased $(20 \%, 40 \%, 60 \%, 80 \%$ and $100 \%)$ and decreased $(-20 \%,-40 \%)$ traffic loads were also considered. The satisfaction probability curves versus traffic loads for the three methods are plotted 

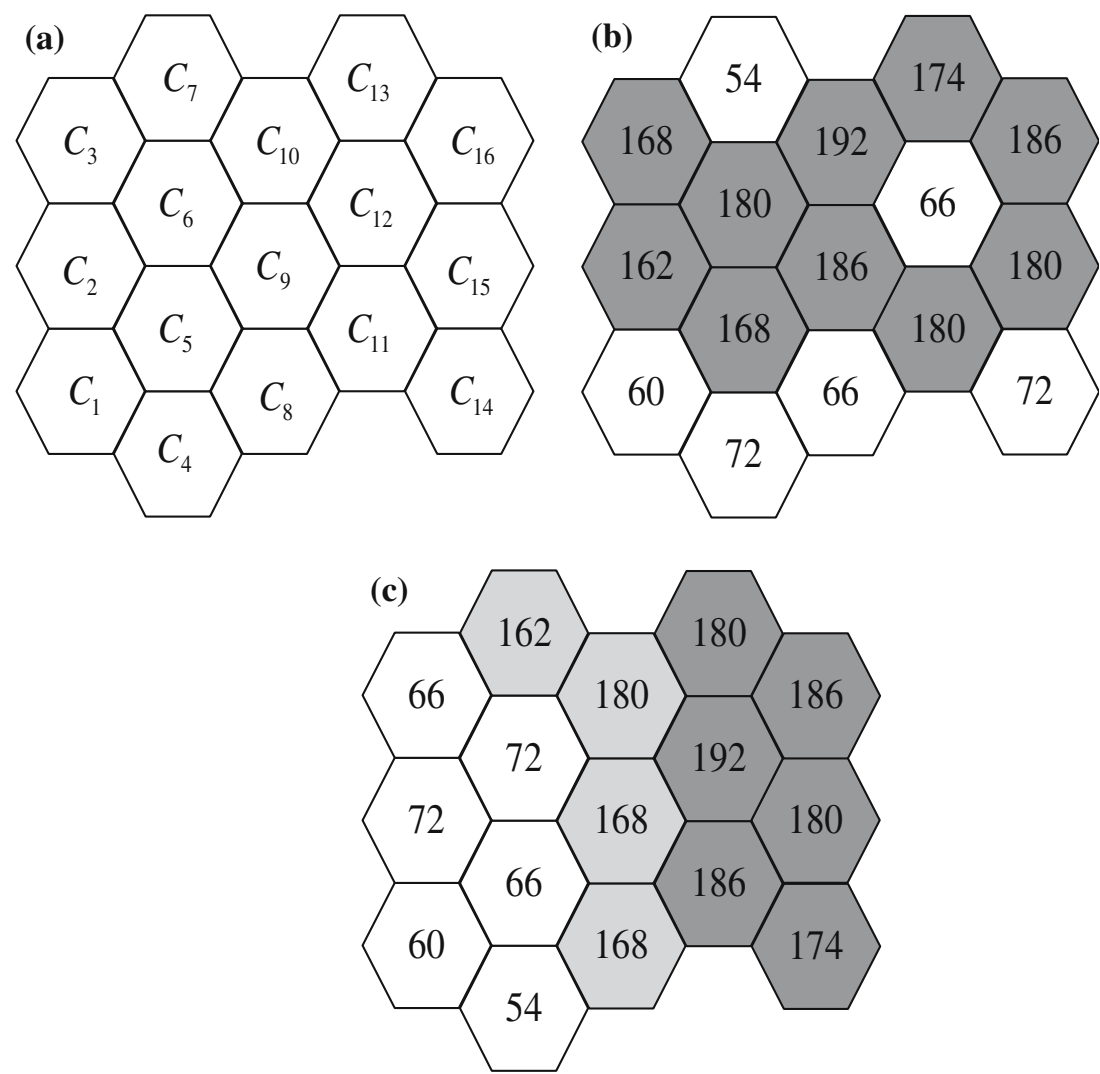

Figure 3. The cellular system used in the simulations. (a) The symbol $C_{i}$ in a cell denotes the $i$ th cell; (b) Nonuniform traffic distribution (calls/h) without patterned traffic load; (c) Nonuniform traffic distribution (calls/h) with patterned traffic load. Light-traffic cells are in the white area. Heavy-traffic cells without neighboring light-traffic cells are in the heavy gray area and heavy-traffic cells with neighboring light-traffic cells are in the light gray area.

in Figure 5(a), where one Erlang means 60 min traffic in a cell. From Figure 5(a), we can see that the proposed method without detection and SB have very close performance and both are much better than FCA. In SB, all the cells can borrow nominal channels from their neighboring cells when all their corresponding nominal channels are currently being used. Compared with FCA, SB makes more nominal channels to be utilized and therefore has much better performance. However, since the underlying traffic is uniform and the re-assignment will not change the equal channel assignment considerably, the proposed method without detection has similar performance to SB.

The second simulation was done by using nonuniform traffic without patterned traffic load. The mean arrival rate is shown in Figure 3(b) and the call duration $\bar{x}$ is still $3 \mathrm{~min}$. The traffic load is that all heavy-traffic cells have at least one light-traffic cell nearby. The light and heavy traffics differ dramatically. However, 

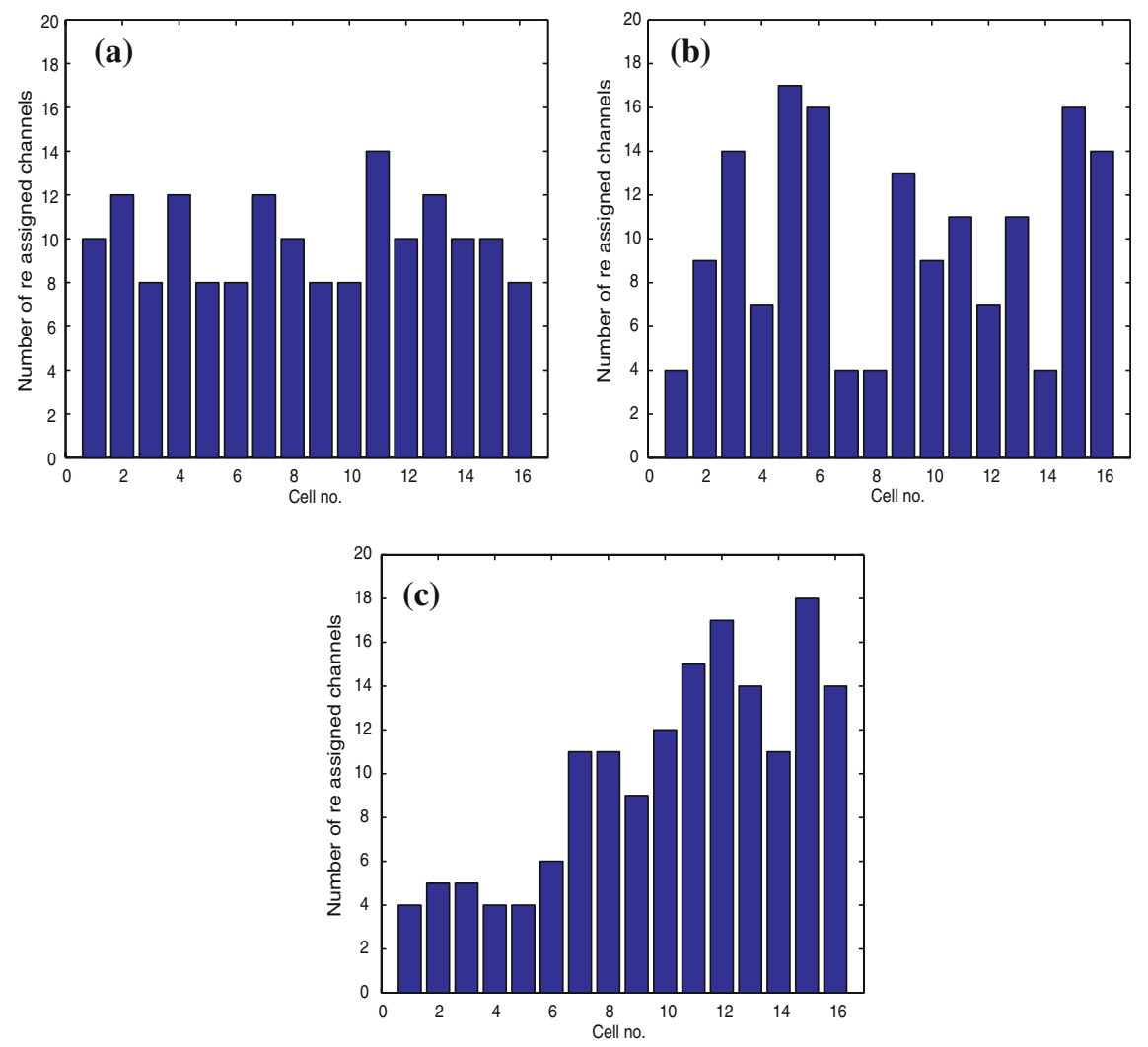

Figure 4. The first channel re-assignment by the proposed method on the 16-cell system under normal condition. (a) Uniform traffic distribution; (b) Nonuniform traffic without patterned traffic load; (c) Nonuniform traffic with patterned traffic load.

a heavy-traffic cell can still borrow a channel from its neighboring light-traffic cells where their nominal channels are not all being used. Since patterned traffic load can seldom happen in this simulation, it was not detected in Step 6 of the proposed method. However, the detection was canceled for later comparison and re-assignment was performed. Under the normal condition without increased or decreased traffic, the first channel re-assignment from the beginning by the proposed method is shown in Figure 4(b). The re-assignment is different from the initial equal assignment at the beginning. It roughly follows the real traffic distribution in Figure 3(b) such that the cells with light traffic are given less nominal channels and those with heavy traffic are given more. The other channel re-assignment at other time is similar to the situation shown in Figure 4(b). Figure 5(b) shows the performance among FCA, SB and the proposed method without detection under different changes of traffic load. For FCA, the light-traffic cells can have some nominal channels never used whilst the heavy-traffic cells can run out of nominal channel at some time. The channel utilization is therefore not good 
for FCA in the second simulation. For SB, a heavy-traffic cell can still have the possibility to borrow a channel from its neighboring light-traffic cells where their nominal channels are not all being used at that time. As expected, SB has better performance than FCA. The performance of the proposed method without detection is very close to that of SB. This is because the traffic in the second simulation is not patterned traffic load and any heavy-traffic cell can still borrow channels from its neighboring light-traffic cells even though the prediction and re-assignment were performed. The advantage of the proposed method cannot be reflected in the second simulation.

The third simulation was conducted by using nonuniform traffic with patterned traffic load. The mean arrival rate is shown in Figure 3(c) and the call duration $\bar{x}$ is still $3 \mathrm{~min}$. The light and heavy traffic also differ dramatically. In the third simulation, patterned traffic load can happen except two extreme cases, i.e., all cells have heavy traffic loads or light traffic loads. However, we also canceled the detection of patterned traffic load in the proposed method for later comparison and the re-assignment was performed. In the third simulation, some heavy-traffic cells (cells 11-16) cannot borrow channels since no light-traffic cells (cells 1-6) are nearby. The number of heavy-traffic cells can occupy about $63 \%$ of the total 16 cells. And about $60 \%$ heavy-traffic cells have no light-traffic neighboring cells. So patterned traffic load can be detected in this simulation. Under the normal condition, the initial channel re-assignment from the beginning by the proposed method is shown in Figure 4(c). The re-assignment is also different from the initial equal assignment at the beginning. It follows the real traffic distribution in Figure 3(c) such that the cells with light traffic are given less nominal channels and those with heavy traffic are given more. The channel re-assignment at other time is similar to the situation shown in Figure 4(c). Under the normal condition, the satisfaction probability for each cell by the proposed method and SB is shown in Figure 6. The satisfaction probabilities of the heavy-traffic cells 11-16, which can hardly borrow channel from their neighboring cells, are increased to some extent by the proposed method. This is due to the increased number of nominal channels for cells $11-16$ by the prediction and re-assignment. The satisfaction probabilities of the heavy-traffic cells $7-10$, which are in the boundary between the heavy-traffic cells and light-traffic cells and can still borrow channels, are similar to those by SB. Since the number of nominal channels after re-assignment for the light-traffic cells is decreased, the performance of the light-traffic cells by the proposed method is a little worse than that by SB. When the average performance is evaluated for the whole system, cells 7-16 are associated with large weights and cells 1-6 are associated with small weights due to the traffic load. And the number of heavytraffic cells is more than that of light-traffic cells. The total effect is that the satisfaction probability for the whole system by the proposed method is higher than that by SB. Figure 5(c) shows the performance among FCA, SB and the proposed method without detection under different changes of traffic load. Similar to the second simulation, both SB and the proposed method are much better than FCA 

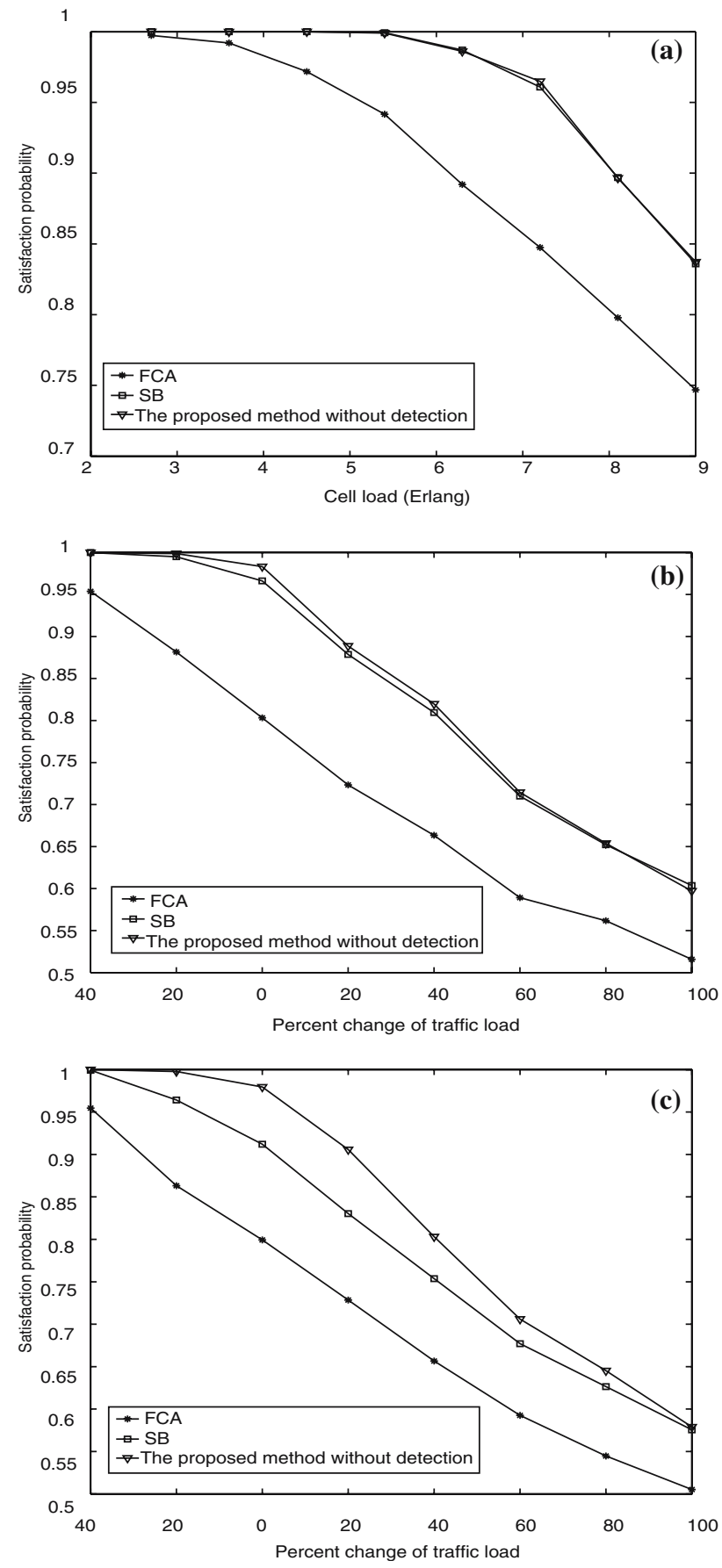

Figure 5. Performance comparison among the three methods. (a) Uniform traffic; (b) Nonuniform traffic without patterned traffic load; (c) Nonuniform traffic with patterned traffic load. 


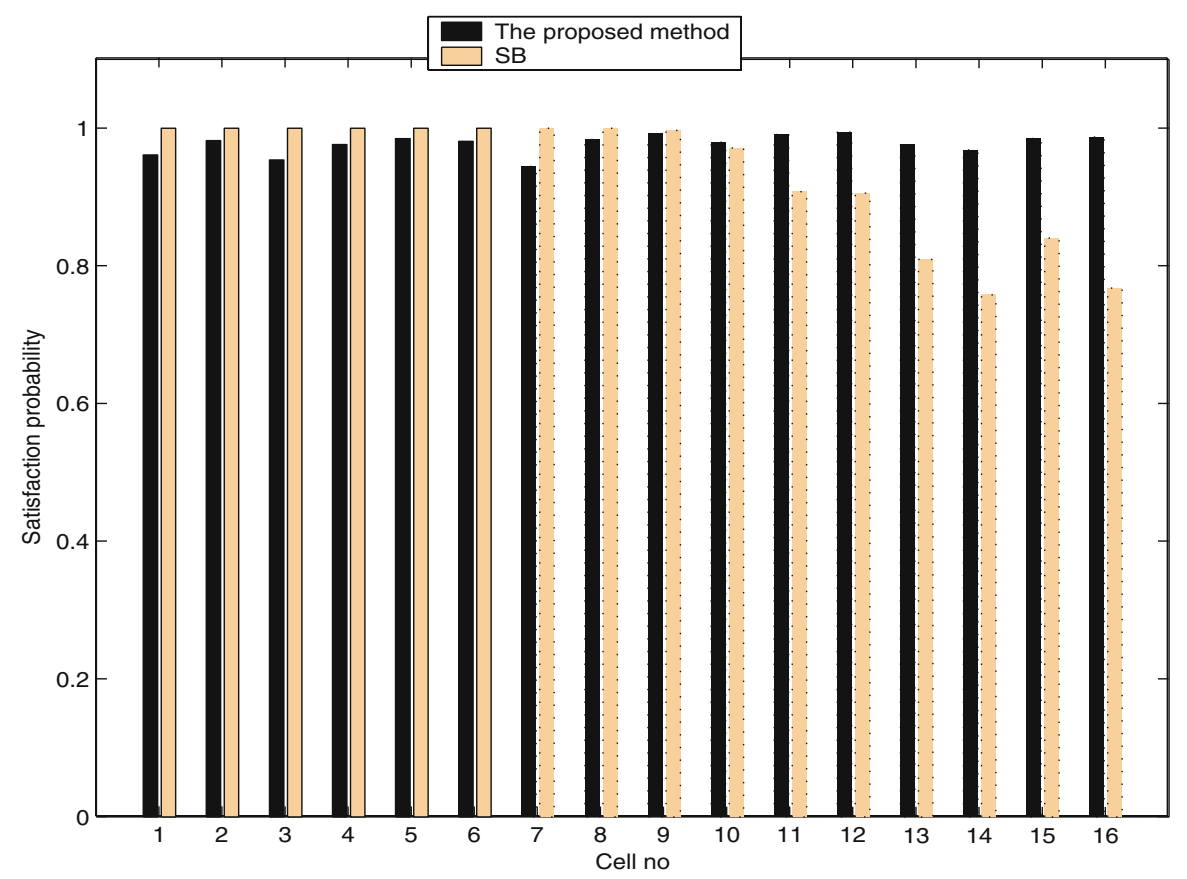

Figure 6. A typical performance comparison between the proposed method and SB for each cell for nonuniform traffic with patterned traffic load. Note that cells 1-6 are light-traffic cells, cells $7-10$ are heavy-traffic cells with neighboring light-traffic cells, and cells 11-16 are heavy-traffic cells without neighboring light-traffic cells.

in the third simulation. The advantage of the proposed method can be reflected here such that the proposed method is better than SB for patterned traffic load by about $3.3 \%, 6.7 \%, 7.5 \%, 5.0 \%$ and $3.0 \%$ increases of satisfaction probability for the traffic increases of $-20 \%, 0 \%, 20 \%, 40 \%$ and $60 \%$, respectively. The improvement is significant because CPSOM directly predicts the traffic to change the initial assignment of SB and does not change the original SB algorithm, which is used directly for channel allocation. In an extreme case when the traffic is not very heavy, i.e., satisfaction probability is above $97 \%$ by SB for $40 \%$ decrease of traffic load, the performance of the proposed method is close to that of SB. This is because patterned traffic load can hardly happen when all cells have light or medium traffic loads and the proposed method provides no improvement for nonpatterned traffic load. On the other extreme (satisfaction probability below $65 \%$ by SB), the performance of the proposed method is also close to that of SB for $80 \%$ and $100 \%$ increases of traffic load. This is also expected since the traffic in each cell is heavy and there is no patterned traffic load in that case. Therefore in case of all cells having heavy traffic, no channels can be borrowed for the proposed method and SB such that they perform in a similar way.

In all the three simulations, the dropping probabilities are all zeros such that all accepted calls are satisfied without dropping. And in all the three simulations, 
the detection of patterned traffic load in the proposed method was canceled for comparison and re-assignment was performed. However, the performance of the proposed method is almost the same as that of SB in traffic situations other than patterned traffic load. Therefore the detection should be always performed to avoid the re-assignment and save computation for non-patterned traffic load. The extra computation of CPSOM and re-assignment in the proposed method can be compensated by a significant improvement of performance for patterned traffic load. The computational complexity of the online CPSOM algorithm with $N$ neurons for handling one input pattern is between $O(N)$ and $O\left(N^{2}\right)$. When $N$ is small, say 9 in this letter, the computation of CPSOM is not heavy.

\section{Conclusions}

In this letter, we consider the situation where many heavy-traffic cells cannot borrow channels from their neighboring cells whilst some light-or-medium-traffic cells still have unused channels. We call this patterned traffic load. This paper shows that we can roughly predict the patterned traffic load by using CPSOM. As a result, we are able to optimize the re-assignment of nominal channels to all cells. Simulations on three temporally stationary traffic loads demonstrate that the proposed method exhibit better performance than SB for patterned traffic load and much better performance than FCA. If other BCA methods are used with CPSOM-based prediction and re-assignment, the results are expected to be similar. When the traffic load is temporally non-stationary and does not vary too rapidly, like the stationary traffic, the proposed method will be better than the traditional FCA and BCA methods if patterned traffic load frequently occurs in the system. Although CPSOM is used directly for traffic prediction and the original BCA method is not altered, the proposed method can deliver better performance than BCA for patterned traffic load situation, and is virtually as good as BCA in the other situations analyzed.

\section{Acknowledgement}

This project is wholly supported by the Hong Kong SAR Government Competitive Earmarked Research Grant 9040803-570.

\section{References}

1. Katzela, I. and Naghshineh, M.: Channel assignment schemes for cellular mobile telecommunication systems: a comprehensive survey, IEEE Personal Communications 3(3) (1996), 10-30.

2. Lee, W. C. Y.: Mobile Cellular Telecommunications Systems, NewYork: McGraw-Hill, 1989.

3. Tamura, H., Sengoku. M., Shinoda, S. and Abe, T.: Channel assignment problem in a cellular system and a new coloring problem of networks, IEICE Transactions on Communications Electronics Information and Systems 74(10) (1991), 2983-2989. 
4. Wang, L., Li, S., Tian, F., and Fu, X.: A noisy chaotic neural network for solving combinatorial optimization problems: stochastic chaotic simulated annealing, IEEE Transactions on Systems, Man, and Cybernetics-Part B: Cybernetics 34(5) (2004), 2119-2125.

5. Kunz, D.: Channel assignment for cellular radio using neural networks, IEEE Transactions on Vehicular Technology 40(1) (1991), 188-193.

6. Funabiki, N. and Takefuji, Y.: A neural network parallel algorithm for channel assignment problems in cellular radio networks, IEEE Transactions on Vehicular Technology 41(4) (1992), 430-437.

7. Smith, K. and Palaniswami, M.: Static and dynamic channel assignment using neural networks, IEEE Transactions on Selected Areas in Communications 15(4) (1997), 238-249.

8. Mathar, R. and Mattfeldt, J.: Channel assignment in cellular radio networks, IEEE Transactions on Vehicular Technology 42(4) (1993), 647-656.

9. Duque-Antón, M., Kunz, D. and Rüber, B.: Channel assignment for cellular radio using simulated annealing, IEEE Transactions on Vehicular Technology 42(1) (1993), 14-21.

10. Capone, A. and Trubian, M.: Channel assignment problem in cellular systems: a new model and a Tabu search algorithm, IEEE Transactions on Vehicular Technology 48(4) (1999), 1252-1260.

11. Montemanni, R., Moon, J. N. J. and Smith, D. H.: An improved Tabu search algorithm for the fixed-spectrum frequency-assignment problem, IEEE Transactions on Vehicular Technology 52(3) (2003), 891-901.

12. Ngo, Y. and Li, V. O. K.: Fixed channel assignment in cellular radio networks using a modified genetic algorithm,IEEE Transactions on Vehicular Technology 47(1) (1998), 163-172.

13. Montemanni, R., Smith, D. H. and Allen, S. M.: An ANTS algorithm for the minimum-span frequency-assignment problem with multiple interference, IEEE Transactions on Vehicular Technology 51(5) (2002), 949-953.

14. Battiti, R., Bertossi, A. and Cavallaro, D.: A randomized saturation degree heuristics for channel assignment in cellular radio networks, IEEE Transactions on Vehicular Technology 50(2) (2001), 364-374.

15. Fernando, X. N. and Fapojuwo, A. O.: A Viterbi-like algorithm with adaptive clustering for channel assignment in cellular radio networks, IEEE Transactions on Vehicular Technology 51(1) (2002), 73-87.

16. Fernando, X. N. and Fapojuwo, A. O.: Cellular radio channel assignment using a sequential trellis search algorithm, In: Vehicular Technology Conference, (VTC 2001), pp. 872-876, Rhodes, Greece, vol. 2, May 2001.

17. Kim, S. and Kim, S.-L.: A two-phase algorithm for frequency assignment in cellular mobile systems, IEEE Transactions on Vehicular Technology 43(3) (1994), 542-548.

18. Funabiki, N., Okutani, N. and Nishikawa, S.: A three-stage heuristic combined neuralnetwork algorithm for channel assignment in cellular mobile systems, IEEE Transactions on Vehicular Technology 49(2) (2000), 397-403.

19. Lázaro, O. and Girma, D.: A Hopfield neural-network-based dynamic channel allocation with handoff channel reservation control, IEEE Transactions on Vehicular Technology 49(5) (2000), 1578-1587.

20. Chan, P. T. H., Palaniswami, M. and Everitt, D.: Neural network-based dynamic channel assignment for cellular mobile communication system, IEEE Transactions on Vehicular Technology 43(2) (1994), 279-288.

21. DelRe, E., Fantacci, R. and Ronga, L.: A dynamic channel allocation technique based on Hopfield neural networks, IEEE Transactions on Vehicular Technology 45(1) (1996), 26-32.

22. Engel, J. S. and Peritsky, M. M.: Statistically-optimum dynamic sever assignment in system with interfering severs, IEEE Transactions on Vehicular Technology 22(4) (1973), 203-209. 
23. Kahwa, T. J. and Georganas, N. D.: A hybrid channel assignment scheme in large-scale cellular-structured mobile communication systems, IEEE Transactions on Communications 26(4) (1978), 432-438.

24. Elnoubi, S. M., Singh, R. and Gupta, S. C.: A new frequency channel assignment algorithm in high capacity mobile communication systems, IEEE Transactions on Vehicular Technology 31(3) (1982), 125-131.

25. Zhang, M. and Yum, T.-K. P.: Comparison of channel-assignment strategies in cellular mobile telephone system, IEEE Transactions on Vehicular Technology 38(4) (1989), 211-215.

26. Chang, K.-N., Kim, J.-T., Yim, C.-S. and Kim, S.: An efficient borrowing channel assignment scheme for cellular mobile systems, IEEE Transactions on Vehicular Technology 47(2) (1998), 602-608.

27. Sandalidis, H. G., Stavroulakis, P. P. and Rodriguez-Tellez, J.: Borrowing channel assignment strategies based on heuristic techniques for cellular systems, IEEE Transactions on Neural Networks 10(1) (1999), 176-181.

28. Chow, T. W. S. and Wu, S.: An online cellular probabilistic self-organizing map for static and dynamic data sets, IEEE Transactions on Circuit and System I 51(4) (2004), 732-747.

29. Kohonen, T.: Self-Organizing Maps, Berlin: Springer, 1997. 